\title{
Design and implementation of a fishery science management information system based on client/server and browser/server models
}

\author{
Hui Fang, Ying Jing, Gang Han and Yingren $\mathrm{Li}^{\star}$ \\ Chinese Academy of Fishery Sciences, Beijing 100141, China. \\ Accepted 14 May, 2013
}

\begin{abstract}
A scientific management information system is the way forward for modern scientific management. The management status and layout of the Chinese Academy of Fishery Sciences is introduced. By comparing the Client/Server and Browser/Server models, a structure combining both models is described together with its network topology. Having analyzed the functional requirements of the management information system, we present a suitable composition of the system. In consideration of the development environment of the system, key technologies are investigated.
\end{abstract}

Key words: Client/Server, Browser/Server, scientific management, information system.

\section{INTRODUCTION}

In recent years, thanks to the rapid development of computer and network technology, the information age has dawned. However, manual management using pen paper and a single type of management, that is, the traditional scientific management model using Word/Excel files, is still widely used. To establish a scientific, systematic, standardized management information system using information technology to reduce the workload, improve efficiency, and promote sharing of research information is the current goal of scientific management (Zhu, 2008; Luo, 2004).

The Chinese Academy of Fishery Sciences (CAFS) is a national fisheries research institution in China, shouldering the responsibility for key basic and applied research and high-tech industrial development. CAFS includes 3 institutes along the coast, 4 institutes situated on rivers, 2 specialty research institutes, as well as 4 fishery resource enhancement stations and the Beijing headquarters. In addition, there are 5 co-built institutes with local governments, located in the 12 provinces (or cities) nationwide. The two-level management structure and geographically wide distribution forces the information in the Chinese Academy of Fishery Sciences to be centralized, making it difficult to integrate and sustain. Therefore, re-planning and organizing scientific management, establishing a networked information system for scientific management to realize "wholeprocess management", improving the quality and efficiency of management, and providing a better service for the Academy, is now an urgent task facing the administrative department of CAFS.

\section{SYSTEM STRUCTURE AND DESIGN}

\section{Client/Server and Browser/Server models}

At present, there are two popular scientific management models: the Client/Server (C/S) and Browser/Server (B/S) models. The $\mathrm{C} / \mathrm{S}$ model consists of two-tiers, connected 


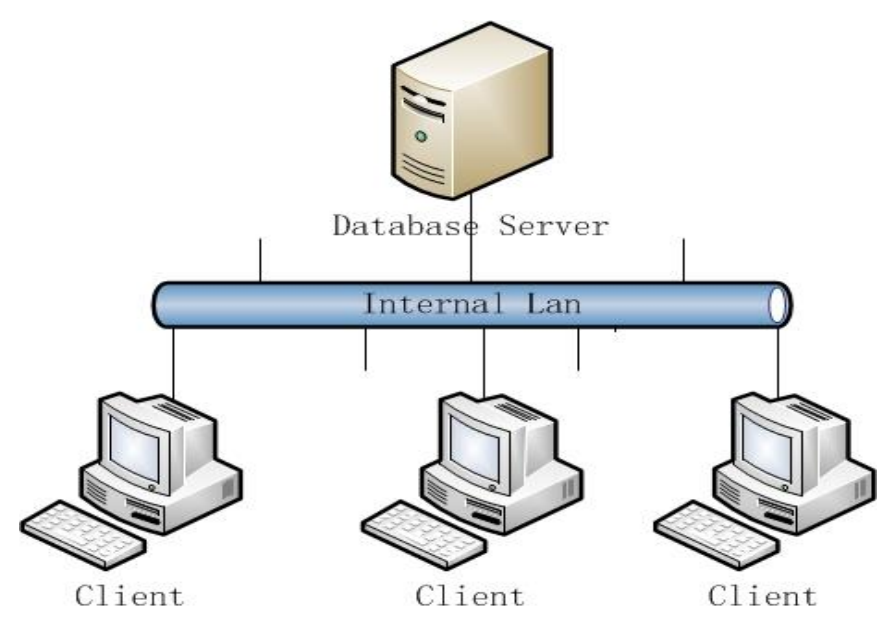

Figure 1. $\mathrm{C} / \mathrm{S}$ mode.

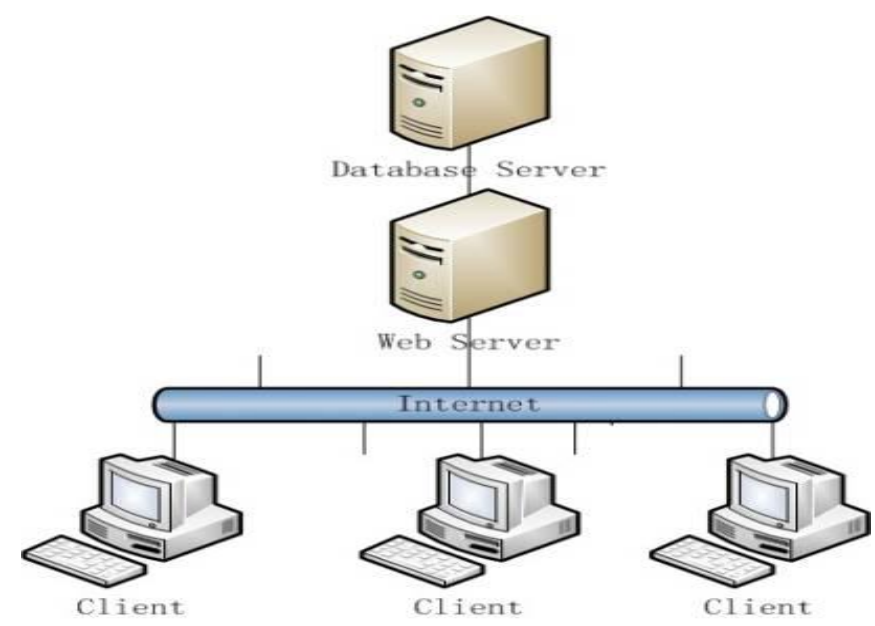

Figure 2. B/S mode.

by a local area network, as shown in Figure 1. It has the advantages of quick processing speed, safe access to data, and small network communication load. However, it is flawed by poor cross-platform service, lack of openness, inconvenient upgrades, redundancy of resources, and so on. The B/S model is Web-based, comprising three tiers, as shown in Figure 2. It offers convenient maintenance and upgrades, consistency of the user interface, as well as openness and good scalability (Wang and Ge, 2006; Liu et al., 2010).

\section{Design of models}

Considering the advantages and disadvantages of the $\mathrm{C} / \mathrm{S}$ and $\mathrm{B} / \mathrm{S}$ models, together with the nature of the scientific management of the CAFS, we adopted a combined $\mathrm{C} / \mathrm{S}$ and $\mathrm{B} / \mathrm{S}$ model to compensate for each model's deficiencies. The choice of using a combined model was made for the following reasons:
1. A scientific management information system should be open-source, scalable, and easy to maintain and upgrade. Since the B/S model is based on general standards accredited by ISO, it is open-source, a general browser is applicable as the Client, and maintenance and upgrades occur at the server side. In addition, since the 3 tiers are independent of each other, they are unaffected by maintenance and expansion in the other tiers (Zhang and Zhao, 2000).

2. The Academy has a 2 tier management structure, but the geographically wide distribution of affiliated institutes makes an internal LAN-based C/S model impracticable. Using the Web based B/S model makes realizing interoperability and data sharing between institutes comparatively easy.

3. However, since the main bodies conducting research work are independent institutes, this facilitates the establishment of separate internal LANs. Therefore, to improve the processing speed of the system, ensure data access security, and reduce the network communication load, the $\mathrm{C} / \mathrm{S}$ model is adopted in each institute.

For the above reasons, a combined $\mathrm{B} / \mathrm{S}$ and $\mathrm{C} / \mathrm{S}$ model is adopted for the Academy's scientific management information system. With this combination, the Web based B/S model used in the Academy and institutes offers the advantages of the Browser/Server model, that is, being light, efficient, and stable, while the C/S model used within the institutes (the research division) contributes the characteristic powerful and high security features. The topology of the information system is depicted in Figure 3.

\section{DESIGN OF SYSTEM FUNCTIONALITY}

\section{Needs of the system}

The underlying principle of the management system is that the process of scientific management should be integrated and statistical analysis should be comprehensive. The overall objective is "the whole process of management" for all research in the Academy, implemented by establishing a network for the scientific management information system. To achieve this objective, the scientific management information system, based on the principle set, should enable unified management of research projects' implementation and cultivation of achievements/awards, while also being able to generate various reports required by clients automatically (Lang et al., 2005).

\section{Modules in the system}

In accordance with the needs of the system, the scientific management information system is divided into 4 modules as depicted in Figure 4: Scientific management, 
Institute's Intranet

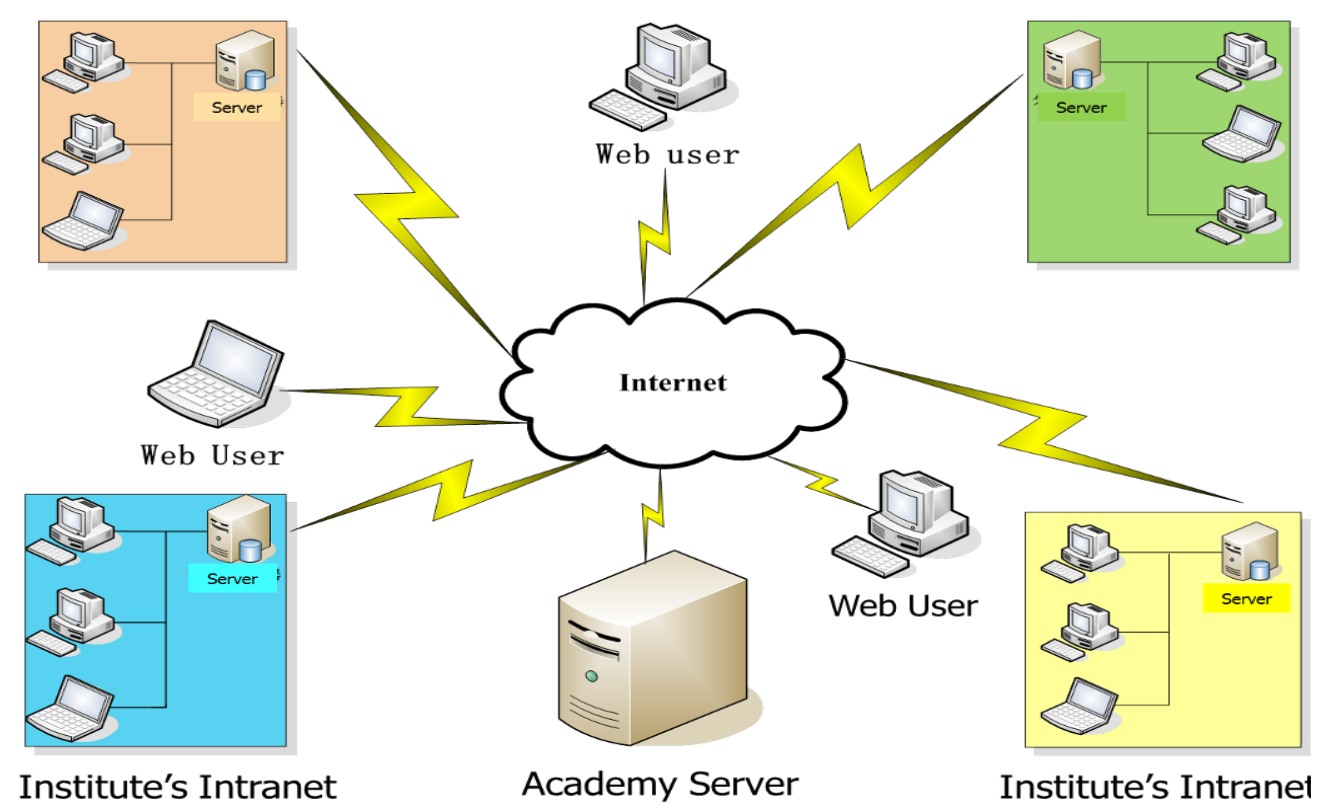

Figure 3. The topology of the information system.

office management, statistics analysis, and maintenance of the system (Ye and Zhang, 2005; Zhu, 2009). The functionality of each module is described below:

(1) Scientific management: to establish the Academy's project library, sci-tech achievements library, equipment library, intellectual property library, publications and papers library, talent databank, and standards databank, and to collect and store the Academy's scientific management related data and information.

(2) Office management: to achieve the daily office management functions, including document registration, leaders' instructions, archiving of records, as well as management of research expenditure, search engine service, printing, and so on.

(3). Statistical analysis: To search and release information by carrying out simple and combined queries on the data within the system, and according to the data selected, to generate various statistical reports, including charts, histograms, and pie charts.

(4) Maintenance of the system: to manage user passwords and access permissions and to import/ export data in a particular format of text or for Excel.

\section{SYSTEM DEVELOPMENT AND KEY TECHNOLOGIES}

\section{Environment for system development}

The environment for developing the scientific management information system is configured as follows:
Microsoft Windows 2000 Professional as the operating system, Microsoft SQL Server 2000 with powerful data processing capabilities as the database management system, Microsoft IIS 5.0 or above as the Web server, Borland Together for VS.NET as a modeling tool, Microsoft Visual SourceSafe for source control management, and Nunit for unit text.

\section{Key technologies for system development}

\section{Design of software architecture}

Due to the fact that the institutes in the Academy are geographically widely distributed, a hierarchical structure for the scientific management information system, distributed top-down in three tiers, has been adopted. The 3 tiers include a user presentation tier, business logic tier, and data tier.

(1) The User Presentation tier provides users with a graphic interface, which helps them understand and position targets efficiently.

(2) The Business Logic Tier implements the application strategies, packages the application modules associated with the system, and presents the packaged module to the user to apply.

(3) The Data Persistence Tier converts the relational database to an object database, allows access to maintain and update data, and realizes communication with the same database server. 


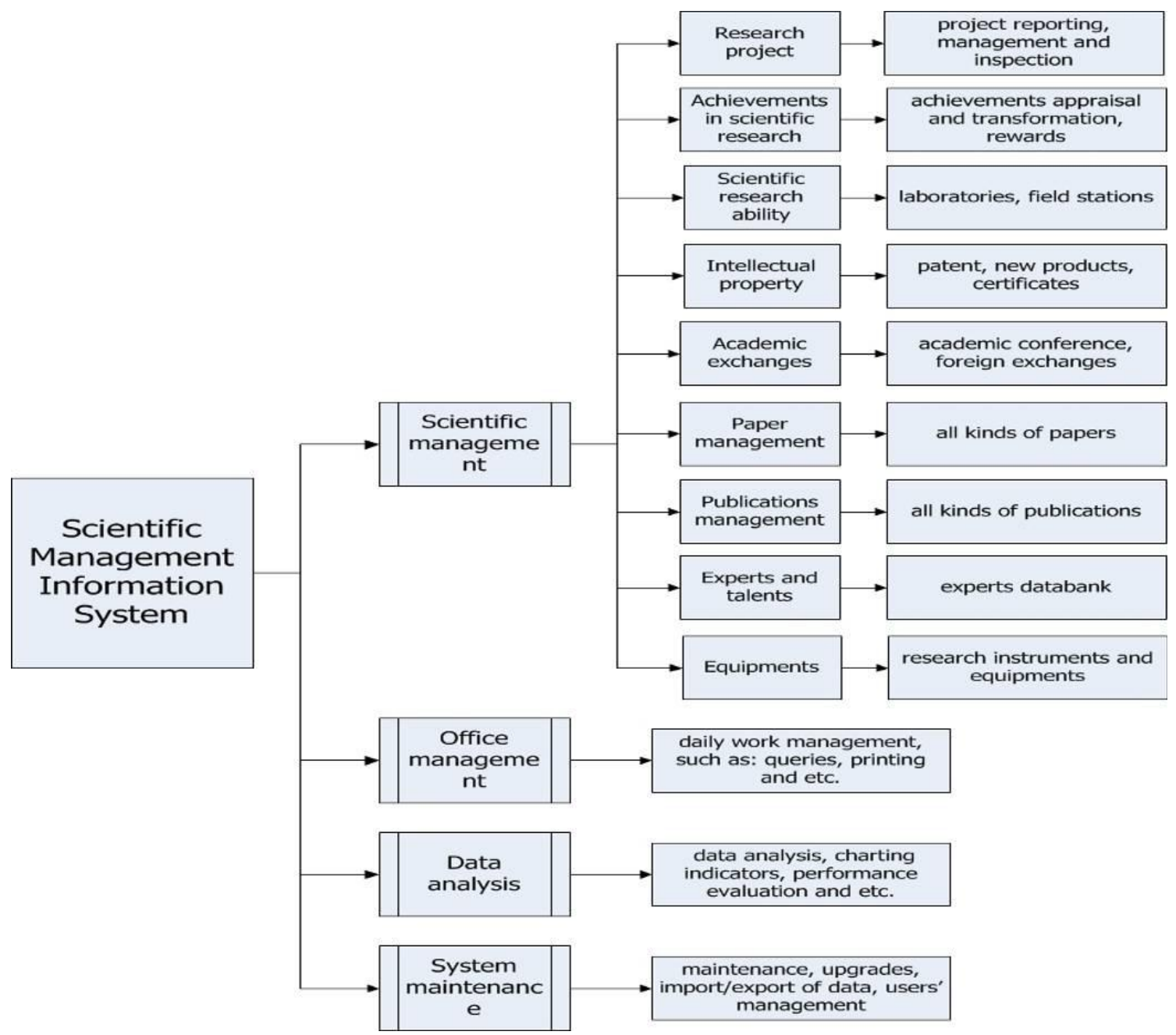

Figure 4. Modules of scientific management information system.

The hierarchical structure above should abate the dependence between tiers. In terms of coding standards, this implies improved reusability of each tier's logic, alleviation of concerns, a loosely coupled system, ability to define standards, and so on. The software architecture is depicted in Figure 5.

\section{Database design and data synchronization}

Ensuring that data within the institutes' databases under the $\mathrm{C} / \mathrm{S}$ model is consistent with that within the Academy's database under the B/S model, is key to the success of the system. To achieve consistency of data at the institute level, ID and InsID are used jointly as the primary key in the data tables under the $\mathrm{C} / \mathrm{S}$ model, of which, the ID is the unique identifier for data in the $\mathrm{C} / \mathrm{S}$ model, while InsID is used to distinguish the source of the data. Meanwhile, the management information system randomly generates a GUID (Globally Unique Identifier) when data is created. This is a character sequence generated automatically, which means that within the same time and space, it is the unique identifier for each computer. In the process of synchronizing the data in the $\mathrm{C} / \mathrm{S}$ and $\mathrm{B} / \mathrm{S}$ models, the system identifies data based on the GUID, and performs the required operations.

Automatic backups and backups by administrators are combined with data synchronization. For important data that needs timely updates, the backup is operated by administrators and synchronized to the Academy's server. All other data is backed-up automatically in accordance with the backup cycle set by the system and synchronized to the Academy's server (Yang and Lin, 2004; Guo et al., 2003).

\section{CONCLUSION}

The aquatic research and management information system presented in this paper is currently operational and running stably. Application of the system has greatly reduced the workload of research managers, significantly 


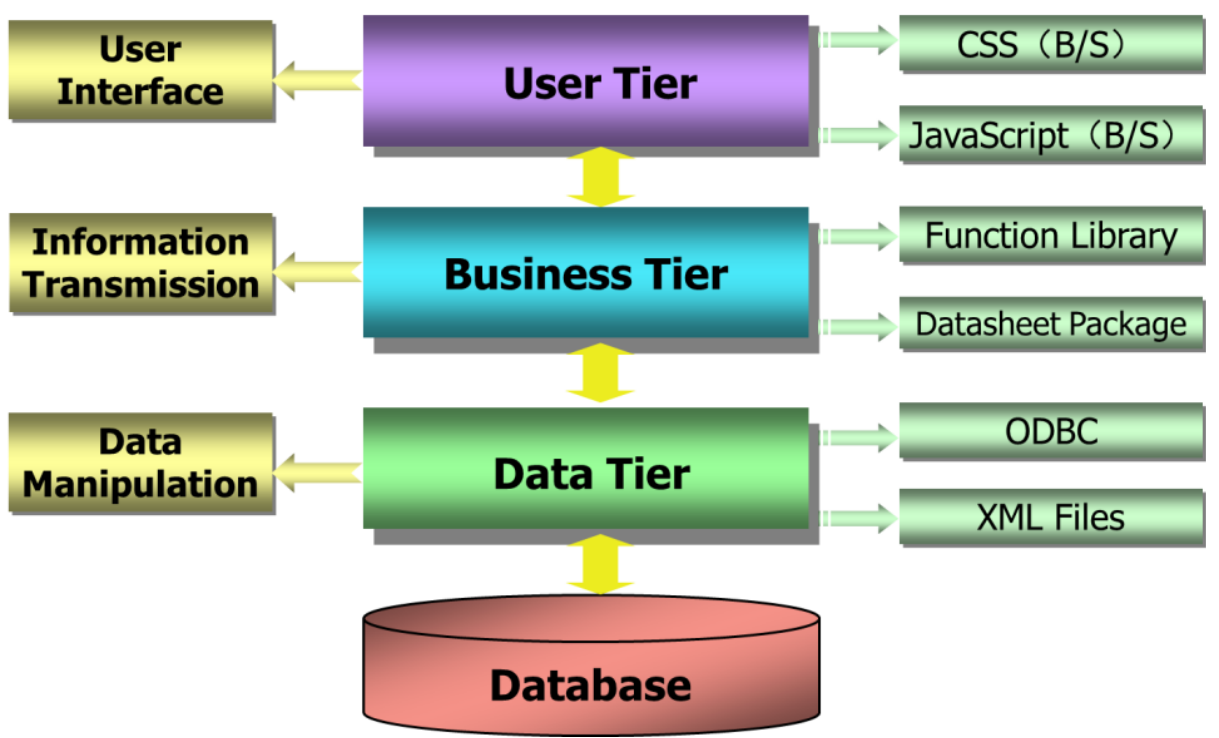

Figure 5. Architecture of the system software.

improved the efficiency and qualifications of scientific management, realized scientification, systematization, standardization, and informationization of the scientific management in the Academy, and provided an important mechanism for scientific management and decisionmaking. It is, therefore, of great value to all users.

\section{REFERENCES}

Guo JY, Shen LZ, Ma GF (2003). Design and Implementation of Collegiate Science Research Management Information System Based on C/S and B/S. Comput. Eng. Application 1:212-214.

Lang Q, Feng L, Xia X (2005). Scientific Management Information System Based on Three- tilter Structure. Comput. Era 5:24-25.

Liu P, Song W, Wan J (2010). Scientific Research Project Management System Based on the Structure of $\mathrm{C} / \mathrm{S}$ Model and B/S Model. Software Guide 9:110-111.

Luo HX (2004). Analysis and Design of the Scientific Research Management Information System Based on Web in Colleges and Universities. Sci. Technol. Manage. Res. 5:122-123.
Wang CD, Ge T (2006). Construction of the Information System for the Management of University's Scientific Researches Based on Internet. J. Anhui Univ. Sci. Technol. Nat. Sci. 1:27-32.

Yang XM, Lin DY (2004). The current status of Management Information Systems. Chinese J. Med. Sci. Res. Manage. 17:219222.

Ye ZW, Zhang XX (2005). The Design and Implement of University Scientific Research Management Information System Based On NET. J. Jiaying Univer. Nat. Sci. 3:71-73.

Zhang XY, Zhao WY (2000). Research and Design of Management Information System of Scientific Research Item. J. Xi'an Univ. Sci. Technol. 20:246-249.

Zhu ZY (2008). The Design and Implementation of Management Information System of University Scientific Research. J. Yancheng Institute. Technol. Natural Sci. Edition. 4:35-37.

Zhu XD (2009). The Construction of Scientific Management Information System. J. Harbin Institute. Technol. Nat. Sci. 11:110-114. 